\title{
MEASURE OF A 2-COMPONENT LINK
}

\author{
JUN O'HARA
}

(Received October 24, 2011, revised February 5, 2013)

\begin{abstract}
A two-component link produces a torus as the product of the component knots in a two-point configuration space of a three-sphere. This space can be identified with a cotangent bundle and also with an indefinite Grassmannian. We show that the integration of the absolute value of the canonical symplectic form is equal to the area of the torus with respect to the pseudo-Riemannian structure, and that it attains the minimum only at the "best" Hopf links.
\end{abstract}

Introduction. Since energy of knots was introduced in [14] about twenty years ago, aiming at producing an optimal knot for each knot type as an energy minimizer, a lot of related works have appeared, which form so-called geometric knot theory (see, for example, $[4,5,16])$. The present paper deals with the same type of topic. We introduce a functional on the space of 2-component links such that the absolute minimum is attained only at "best" Hopf links, not at trivial links.

Let $C_{1} \cup C_{2}$ be a 2-component link in $S^{3}$. The value of our functional $A\left(C_{1}, C_{2}\right)$ can be interpreted in the following two ways. Observe that the link produces a torus $C_{1} \times C_{2}$ in $S^{3} \times S^{3} \backslash \Delta$, where $\Delta$ is the diagonal set.

First, there is a natural identification between $S^{3} \times S^{3} \backslash \Delta$ and the total space of the cotangent bundle $T^{*} S^{3}$. The pull-back $\omega$ of the canonical symplectic form of $T^{*} S^{3}$ to $S^{3} \times S^{3} \backslash \Delta$ is the unique 2-form (up to multiplication by a constant) which is invariant under the diagonal action of the Möbius group. The 2-form $\omega$ can also be considered as a natural symplectic form on the space of geodesics in a hyperbolic 4-space $H^{4}$. As $\omega$ is exact, $\int_{C_{1} \times C_{2}} \omega$ vanishes, but $\int_{C_{1} \times C_{2}}|\omega|$ does not, which is $A\left(C_{1}, C_{2}\right)$. In this sense, it can be considered as an "absolute symplectic measure" of the torus $C_{1} \cup C_{2}$ in $T^{*} S^{3}$.

Second, from a Möbius geometric viewpoint, $S^{3} \times S^{3} \backslash \Delta$ can be identified with the Grassmannian manifold $S O(4,1) / S O(1,1) \times S O(3)$ of oriented time-like 2-dimensional vector subspaces in the 5-dimensional Minkowski space $\boldsymbol{R}_{1}^{5}$. By taking a pseudo-orthogonal complement of an oriented time-like 2-dimensional vector subspace, we can identify this space with the Grassmannian manifold of oriented space-like 3-dimensional vector subspaces in $\boldsymbol{R}_{1}^{5}$.

It has a natural pseudo-Riemannian structure which is compatible with the action of the Lorentz group, which induces the diagonal action of the Möbius group to $S^{3} \times S^{3} \backslash \Delta$. Then

2000 Mathematics Subject Classification. Primary 57M25; Secondary 53A30.

Key words and phrases. Energy, link, symplectic measure, Möbius geometry, pseudo-Riemannian geometry.

Partly supported by the Grant-in-Aid for Scientific Research (C), Japan Society for the Promotion of Science. 
$A\left(C_{1}, C_{2}\right)$ is equal to the measure (area) of the torus $C_{1} \cup C_{2}$ with respect to the pseudoRiemannian metric.

The key of the proof is that both the pull-back $\omega$ of the canonical symplectic form and the "imaginary signed area element" with respect to the pseudo-Riemannian structure coincide with the real part of the infinitesimal cross ratio, which is a "complex valued 2-form" on $C_{1} \times C_{2}$ used in the joint paper with Langevin [12]. Geometrically, it can be considered as the cross ratio of $x, x+d x, y$ and $y+d y$, where these four points are considered as complex numbers by identifying a sphere through them with the Riemann sphere $C \cup\{\infty\}$.

Some remarks on the result on the energy of links [1], which is another characterization of the "best" Hopf link, will be given in Subsection 4.4.

Throughout the paper, a link means a smooth (or at least of class $C^{1}$ ) 2-component link.

Acknowledgment. The author thanks deeply Rémi Langevin, Masahiko Kanai and Luisa Paoluzzi for helpful suggestions. He also thanks the referee for a helpful suggestion concerning the symplectic form.

1. Two structures on $S^{3} \times S^{3} \backslash \Delta$. We introduce two structures on $S^{3} \times S^{3} \backslash \Delta$, the symplectic structure and the pseudo-Riemannian structure, both compatible with Möbius transformations. It is easy to see that both can be naturally generalized to $S^{n} \times S^{n} \backslash \Delta$ for any $n$.

\subsection{Symplectic structure of $S^{3} \times S^{3} \backslash \Delta$.}

1.1.1. Via hyperbolic space. As $S^{3}$ can be considered as the boundary of 4-dimensional hyperbolic space $H^{4}, S^{3} \times S^{3} \backslash \Delta$ can be considered as the space of oriented geodesics in $H^{4}$, which is denoted by $\mathcal{G}$. The tangent space $T_{\gamma} \mathcal{G}$ along a geodesic $\gamma$ is the space of Jacobi fields along $\gamma$. Let $\nabla$ denote the Levi-Civita connection. Then, if we put

$$
\omega_{g}(\xi, \eta)=\left(\xi(t), \nabla_{\dot{\gamma}} \eta(t)\right)-\left(\eta(t), \nabla_{\dot{\gamma}} \xi(t)\right) \quad(t \in \boldsymbol{R})
$$

for $\xi, \eta \in T_{\gamma} \mathcal{G}$, where (, ) denotes the standard inner product on $T_{\gamma(t)} H^{4}$, then $\omega_{g}$ is an isometry-invariant symplectic form on $\mathcal{G}$ (see $[3,2 \mathrm{C}],[11,3.1]$ ). Since an isometry of $H^{4}$ induces a Möbius transformation of the boundary sphere $S^{3}, \omega_{g}$ defines a symplectic form on $S^{3} \times S^{3} \backslash \Delta$ which is invariant under the diagonal action of the Möbius group.

1.1.2. Via cotangent bundle. It is known that the space $\mathcal{G}$ of geodesics in $H^{4}$ is symplectomorphic to the cotangent bundle $T^{*} S^{3}$ [6]. Let us give an identification between $S^{3} \times S^{3} \backslash \Delta$ and $T^{*} S^{3}$ explicitly.

Assume $S^{3}$ is the unit sphere in $\boldsymbol{R}^{4}$. Let $x$ be a point in $S^{3}$ and $p_{x}: S^{3} \backslash\{x\} \rightarrow$ $(\operatorname{Span}\langle x\rangle)^{\perp}$ be a stereographic projection. By identifying $(\operatorname{Span}\langle x\rangle)^{\perp}$ with $T_{x} S^{3} \cong T_{x}^{*} S^{3}$, we obtain a bijection

$$
\varphi_{x}: S^{3} \backslash\{x\} \ni y \mapsto\left(T_{x} S^{3} \ni v \mapsto p_{x}(y) \cdot v \in \boldsymbol{R}\right) \in T_{x}^{*} S^{3},
$$

where $\cdot$ denotes the standard inner product in $\boldsymbol{R}^{4}$. It induces a bijection

$$
\varphi: S^{3} \times S^{3} \backslash \Delta \ni(x, y) \mapsto\left(x, \varphi_{x}(y)\right) \in T^{*} S^{3} .
$$


Let $\omega_{S^{3}}$ be the canonical symplectic form of the cotangent bundle $T^{*} S^{3}$. Put $\omega=\varphi^{*} \omega_{S^{3}}$. In [12], we showed that $\omega$ is invariant under the diagonal action of the Möbius group. The converse is also true. Namely, if a 2-form $\rho$ is invariant under the diagonal action of the Möbius group, then $\rho=c \omega$ for some $c \in \boldsymbol{R}$ (Proposition 4.1 in Appendix). Therefore, we can see that $\omega$ coincides with $\omega_{g}$ mentioned above up to a constant factor.

1.2. Pseudo-Riemannian structure of $S^{3} \times S^{3} \backslash \Delta$. The Minkowski space $\boldsymbol{R}_{1}^{5}$ is $\boldsymbol{R}^{5}$ with the indefinite inner product

$$
\langle x, y\rangle=-x_{0} y_{0}+x_{1} y_{1}+\cdots+x_{4} y_{4} .
$$

The set of light-like vectors and the origin $L=\left\{v \in \boldsymbol{R}_{1}^{5} ;\langle v, v\rangle=0\right\}$ is called the light cone. The 3 -sphere can be considered as the projectivization $\boldsymbol{P} L$ of the light cone. It can also be identified isometrically with the intersection of the light cone and a hyperplane given by $\{x ;\langle x, n\rangle=-1\}$, where $n$ is a unit time-like vector. A 2-dimensional vector subspace $\Pi$ of $\boldsymbol{R}_{1}^{5}$ is said to be time-like if $\left.\langle\rangle\right|_{,\Pi}$ is non-degenerate and indefinite, namely, if $\Pi$ intersects the light cone transversely.

A pair of points in $S^{3}$ can be considered as the intersection of $S^{3}$ and a 2-dimensional time-like subspace of $\boldsymbol{R}_{1}^{5}$. Therefore, if we also take the order of the points into account, $S^{3} \times S^{3} \backslash \Delta$ can be identified with the Grassmannian manifold $\widetilde{G r}_{-}\left(2 ; \boldsymbol{R}_{1}^{5}\right)$ of oriented 2dimensional time-like subspaces of $\boldsymbol{R}_{1}^{5}$, i.e., a homogeneous space $S O(4,1) / S O(3) \times$ $S O(1,1)$.

Let $\Pi$ be an oriented time-like 2-dimensional plane spanned by an ordered basis $\{u, v\}$. Then $\Pi$ corresponds to a pure 2-vector $u \wedge v \in \bigwedge^{2} \boldsymbol{R}_{1}^{5}$, which is determined by $\Pi$ up to a positive factor. As is stated on page 280 of [9], $u \wedge v$ is time-like, i.e., $\langle u \wedge v, u \wedge v\rangle<0$, where the indefinite inner product on $\bigwedge^{2} \boldsymbol{R}_{1}^{5}$ is given by

$$
\left\langle u^{1} \wedge u^{2}, v^{1} \wedge v^{2}\right\rangle=\operatorname{det}\left(\left\langle u^{i}, v^{j}\right\rangle\right) .
$$

On the other hand, it is known that a pure 2-vector determines a 2-plane. Thus the Grassmannian manifold $\widetilde{\mathrm{Gr}}_{-}\left(2 ; \boldsymbol{R}_{1}^{5}\right)$ of oriented 2-dimensional time-like subspaces of $\boldsymbol{R}_{1}^{5}$ can be identified with the set of unit time-like pure 2-vectors in $\bigwedge^{2} \boldsymbol{R}_{1}^{5}$, where the norm of $\bigwedge^{2} \boldsymbol{R}_{1}^{5}$ is given by $\|v\|=\sqrt{|\langle v, v\rangle|}$. It is a 6-dimensional pseudo-Riemannian manifold with index 3. By taking a pseudo-orthogonal complement of an oriented time-like 2-dimensional vector subspace, we can identify $\widetilde{\mathrm{Gr}}_{-}\left(2 ; \boldsymbol{R}_{1}^{5}\right)$ with the Grassmannian manifold $\widetilde{\mathrm{Gr}}_{+}\left(3 ; \boldsymbol{R}_{1}^{5}\right)$ of oriented space-like 3-dimensional vector subspaces in $\boldsymbol{R}_{1}^{5}$, which, in turn, can be identified with the set $\Theta(0,3)$ of unit space-like pure 3-vectors in $\bigwedge^{3} \boldsymbol{R}_{1}^{5}$.

Through the identifications mentioned above, the bijection from $\widetilde{\mathrm{Gr}}_{-}\left(2 ; \boldsymbol{R}_{1}^{5}\right)$ to $\widetilde{G r}_{+}\left(3 ; R_{1}^{5}\right)$ is equal to the minus of the restriction of the Hodge $\star$ which is an isomorphism from $\bigwedge^{2} \boldsymbol{R}_{1}^{5}$ to $\bigwedge^{3} \boldsymbol{R}_{1}^{5}$ given by

$$
a \wedge \star b=\langle a, b\rangle e_{0} \wedge e_{1} \wedge \cdots \wedge e_{4} \quad\left(a, b \in \bigwedge^{2} \boldsymbol{R}_{1}^{5}\right)
$$


(see [9, p. 288]).

Let $u$ and $v$ be light-like vectors in $\boldsymbol{R}_{1}^{5}$. Put $u \times v=-\star(u \wedge v) \in \bigwedge^{3} \boldsymbol{R}_{1}^{5}$. Since the Hodge $\star$ satisfies $\langle\star a, \star b\rangle=-\langle a, b\rangle$, where $a, b \in \stackrel{2}{\bigwedge} \boldsymbol{R}_{1}^{5}$, we have

$$
\left\langle u^{1} \times u^{2}, v^{1} \times v^{2}\right\rangle=-\operatorname{det}\left(\left\langle u^{i}, v^{j}\right\rangle\right) .
$$

Thus we have a bijection

$$
\psi: S^{3} \times S^{3} \backslash \Delta \ni(x, y) \mapsto \frac{x \times y}{\|x \times y\|} \in \Theta(0,3) .
$$

Since the indefinite inner product in (2) is invariant under the action of the Lorentz group $O(4,1)$, the pseudo-Riemannian structure on $S^{3} \times S^{3} \backslash \Delta$ induced by $\psi$ is invariant under the diagonal action of the Möbius group.

2. Measure of a 2-component link. All the pairs of points $\left\{(x, y) ; x \in C_{1}, y \in C_{2}\right\}$ form a torus in $S^{3} \times S^{3} \backslash \Delta$. Let us call it the product torus of a 2-component link $L=C_{1} \cup C_{2}$.

2.1. Area of the product torus of a link. Let $\sigma$ be the composite of maps:

$$
\sigma: C_{1} \times C_{2} \stackrel{\iota}{\hookrightarrow} S^{3} \times S^{3} \backslash \Delta \stackrel{\cong}{\underset{\psi}{\cong}} \Theta(0,3) .
$$

We identify $\sigma\left(C_{1} \times C_{2}\right)$ with $C_{1} \times C_{2}$ in what follows. The area element $d v$ of $C_{1} \times C_{2}$ associated with the pseudo-Riemannian structure of $\Theta(0,3)$ is given by

$$
d v=\sqrt{\left|\operatorname{det}\left(\begin{array}{cc}
\left\langle\sigma_{x}, \sigma_{x}\right\rangle & \left\langle\sigma_{x}, \sigma_{y}\right\rangle \\
\left\langle\sigma_{y}, \sigma_{x}\right\rangle & \left\langle\sigma_{y}, \sigma_{y}\right\rangle
\end{array}\right)\right|} d x \wedge d y
$$

where $\sigma_{x}$ and $\sigma_{y}$ denote $\partial \sigma / \partial x(x, y)$ and $\partial \sigma / \partial y(x, y)$ in $T_{\sigma(x, y)} \Theta(0,3)$, respectively.

Definition 2.1. Define the measure of a 2-component link $L=C_{1} \cup C_{2}$ by the area of the product torus

$$
A\left(C_{1}, C_{2}\right)=\int_{C_{1} \times C_{2}} d v=\int_{C_{1} \times C_{2}} \sqrt{\left|\operatorname{det}\left(\begin{array}{cc}
\left\langle\sigma_{x}, \sigma_{x}\right\rangle & \left\langle\sigma_{x}, \sigma_{y}\right\rangle \\
\left\langle\sigma_{y}, \sigma_{x}\right\rangle & \left\langle\sigma_{y}, \sigma_{y}\right\rangle
\end{array}\right)\right|} d x \wedge d y .
$$

\subsection{Main Theorem.}

THEOREM 2.2. (i) The measure of a 2-component link satisfies

$$
A\left(C_{1}, C_{2}\right)=\int_{C_{1} \times C_{2}}\left|\iota^{*} \omega\right|,
$$

where $\iota$ is the inclusion from $C_{1} \times C_{2}$ into $S^{3} \times S^{3} \backslash \Delta$ and $\omega$ is the pull-back of the canonical symplectic form of $T^{*} S^{3}$ to $S^{3} \times S^{3} \backslash \Delta$.

(ii) The measure of a 2-component link takes its minimum value 0 if and only if $L$ is the image of the "best" Hopf link

$$
\left\{(z, w) \in C^{2} ;|z|=1, w=0\right\} \cup\left\{(z, w) \in C^{2} ; z=0,|w|=1\right\} \subset S^{3}
$$

by a Möbius transformation. 
The equation (4) implies that the area of the product torus can also be called the "absolute symplectic measure" of it.

We prove the theorem in the next section.

2.3. Area element of a product torus in $S^{3} \times S^{3} \backslash \Delta$.

LEMMA 2.3. Both $\sigma_{x}$ and $\sigma_{x}$ are null vectors, i.e., $\left\langle\sigma_{x}, \sigma_{x}\right\rangle=\left\langle\sigma_{y}, \sigma_{y}\right\rangle=0$. Therefore the area element $d v$ is given by $\sigma^{*} d v=\left|\left\langle\sigma_{x}, \sigma_{y}\right\rangle\right| d x \wedge d y$.

Proof. Suppose $S^{3}$ is embedded in $\boldsymbol{R}_{1}^{5}$, and points in $C_{1}$ and $C_{2}$ are expressed by $\bar{x}(s)$ and $\bar{y}(t)$, respectively. Put $p(s, t)=\bar{x}(s) \times \bar{y}(t)$ and $\tilde{\sigma}(s, t)=\sigma(\bar{x}(s), \bar{y}(t))$. Then it is given by

$$
\tilde{\sigma}(s, t)=\frac{p(s, t)}{\langle p(s, t), p(s, t)\rangle^{1 / 2}} .
$$

Since $\bar{x}$ and $\bar{y}$ are light-like vectors, the formula (2) implies

$$
\langle p, p\rangle=\langle\bar{x}, \bar{y}\rangle^{2},\left\langle p, p_{s}\right\rangle=\langle\bar{x}, \bar{y}\rangle\left\langle\bar{x}_{s}, \bar{y}\right\rangle,\left\langle p_{s}, p_{s}\right\rangle=\left\langle\bar{x}_{s}, \bar{y}\right\rangle^{2} .
$$

Therefore

$$
\left\langle\tilde{\sigma}_{s}, \tilde{\sigma}_{s}\right\rangle=\frac{\langle p, p\rangle\left\langle p_{s}, p_{s}\right\rangle-\left\langle p, p_{s}\right\rangle^{2}}{\langle p, p\rangle^{2}}=0 .
$$

We also put geometric explanation in Subsection 4.2 in Appendix.

Let us call $\left\langle\sigma_{x}, \sigma_{y}\right\rangle d x \wedge d y$ the imaginary signed area element of a product torus $C_{1} \times C_{2}$.

\section{Proof of the main theorem.}

3.1. The infinitesimal cross ratio. We assume that both components $C_{1}$ and $C_{2}$ are oriented. Suppose $x \in C_{1}$ and $y \in C_{2}$. Let $\Gamma(x, x, y)$ be the circle which is tangent to $C_{1}$ at $x$ that passes through $y$, oriented by the tangent vectors to $C_{1}$ at $x$. Let $\theta(0 \leq \theta \leq \pi)$ be the angle between $\Gamma(x, x, y)$ and the tangent vector to $C_{2}$ at $y$. We call it the conformal angle between $x$ and $y$ and denote it by $\theta_{L}(x, y)$. It was introduced by Doyle and Schramm.

Let $\Omega_{L}$ be a complex valued 2-form on $C_{1} \times C_{2}$ given by

$$
\Omega_{L}(x, y)=e^{i \theta_{L}(x, y)} \frac{d x \wedge d y}{|x-y|^{2}}
$$

(see [12]). As both the conformal angle $\theta_{L}$ and the 2-form $d x d y /|x-y|^{2}$ are equivariant under the diagonal action of a Möbius transformation $T$, so is $\Omega_{L}$, namely, $(T \times T)^{*} \Omega_{T(L)}=$ $\Omega_{L}[12]$.

Let us give a geometric interpretation of $\Omega_{L}$. Let $\Sigma_{L}(x, y)$ be a sphere that passes through four points $x, x+d x, y$ and $y+d y$, i.e., a sphere which is tangent to $C_{1}$ at $x$ and to $C_{2}$ at $y$. Let $p$ be a stereographic projection from $\Sigma_{L}(x, y)$ to $C \cup\{\infty\}$ and $\tilde{x}, \tilde{x}+\widetilde{d x}, \tilde{y}$ and $\tilde{y}+\widetilde{d y}$ the images by $p$ of the four points $x, x+d x, y$ and $y+d y$, respectively. Then $\Omega_{L}(x, y)$ is equal to the cross ratio $(\tilde{x}+\widetilde{d x}, \tilde{y} ; \tilde{x}, \tilde{y}+\widetilde{d y})$ :

$$
\Omega_{L}(x, y)=\frac{\widetilde{d x} \widetilde{d y}}{(\tilde{x}-\tilde{y})^{2}} \sim \frac{(\tilde{x}+\widetilde{d x})-\tilde{x}}{(\tilde{x}+\widetilde{d x})-(\tilde{y}+\widetilde{d y})}: \frac{\tilde{y}-\tilde{x}}{\tilde{y}-(\tilde{y}+\widetilde{d y})} .
$$


This is why we call $\Omega_{L}$ the infinitesimal cross ratio. We remark that the cross ratio does not depend on the stereographic projection $p$.

REMARK 3.1. The form $d z d w /(z-w)^{2}$ on $\boldsymbol{C} \times \boldsymbol{C} \backslash \Delta$, which has been used in complex analysis, can also be obtained as the cross ratio of $w, w+d w, z$ and $z+d z$, as was mentioned by Rob Kusner, for example. In this sense, the infinitesimal cross ratio can be considered as generalization of $d z d w /(z-w)^{2}$ to a complex valued 2-form on $C_{1} \times C_{2}$, or in general, $C \times C \backslash \Delta$, where $C$ is a union of space curves. In fact, when $C$ is a plane curve, the infinitesimal cross ratio coincides up to complex conjugacy with the 2-form that is obtained by restricting $d z d w /(z-w)^{2}$ to $C \times C \backslash \Delta$, which was used by Hélein [8] to show the isoperimetric inequality.

However, there is difficulty for space curves. First, $d z d w /(z-w)^{2}$ cannot be generalized to a 2 -form on the ambient space $S^{3} \times S^{3} \backslash \Delta$, so the restriction which works for the planar case does not work. To be precise, while the real part of $d z d w /(z-w)^{2}$ can be generalized to a 2-form on $S^{n} \times S^{n} \backslash \Delta$ as we will see in the next subsection, the imaginary part cannot when $n \geq 3$ as we will see in Proposition 4.1.

Secondly, even if we try to use the cross ratio to define the 2-form, the cross ratio of four points in $\boldsymbol{R}^{n}(n \geq 3)$ is not so well-behaved as in the planar case. This might be a reason why Ahlfors studied only the absolute cross ratio for the points in $\boldsymbol{R}^{n}(n \geq 3)$ [2]. When we want to define the cross ratio of (ordered) four points in $\boldsymbol{R}^{3}$, we need the orientation of the sphere through the four points to avoid the ambiguity of complex conjugacy. There is a way to assign continuously the orientations to all the spheres given by the sets of ordered four points in $\boldsymbol{R}^{3}$, i.e., there is a continuous map from $\left(\boldsymbol{R}^{3}\right)^{4} \backslash \Delta$, where $\Delta$ is a big diagonal set, to the set of oriented 2-speres in $\boldsymbol{R}^{3}$, which can be identified with the de Sitter space in 5-dimensional Minkowski space $\boldsymbol{R}_{1}^{5}$. However, according to this method, the imaginary part of the cross ratio of any four points in $\boldsymbol{R}^{3}$ is always non-negative (or, always non-positive according to the choice of a continuous map from $\left(\boldsymbol{R}^{3}\right)^{4} \backslash \Delta$ to the de Sitter space). The reader is referred to [15] for the details. As a result, the imaginary part of the infinitesimal cross ratio may have singularity where it vanishes, just like that of the absolute value of a smooth function. Anyway, we do not use the imaginary part in this paper.

3.2. The real part of the infinitesimal cross ratio. In [12] we showed that the pullback of the canonical symplectic form of $T^{*} S^{3}$ to $C_{1} \times C_{2}$ coincides with the real part of the infinitesimal cross ratio up to a constant;

$$
\iota^{*} \omega=\iota^{*} \varphi^{*} \omega_{S^{3}}=-2 \Re e \Omega_{L}=-2 \frac{\cos \theta_{L}(x, y) d x \wedge d y}{|x-y|^{2}} .
$$

It seems that this fact in the case of $S^{2}$ is well known in symplectic geometry.

LEMMA 3.2. The imaginary signed area element of $C_{1} \times C_{2}$ with respect to the pseudoRiemannian structure coincides with the real part of the infinitesimal cross ratio up to a constant;

$$
\left\langle\sigma_{x}, \sigma_{y}\right\rangle d x \wedge d y=2 \Re \mathfrak{R} \Omega_{L} .
$$




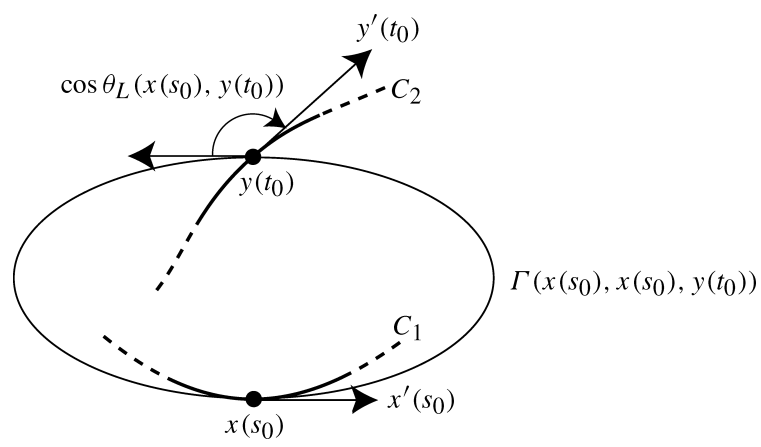

FIGURE 1.

Proof. Suppose points in $C_{1}$ and $C_{2}$ are expressed as $x(s)$ and $y(t)$. Suppose $S^{3}$ is embedded in $\boldsymbol{R}_{1}^{5}$ as the intersection of the light cone and a level hyperplane $\left\{x_{0}=1\right\}$. Let $\bar{x}$ and $\bar{y}$ be points in $\boldsymbol{R}_{1}^{5}$ corresponding to $x(s)$ and $y(t)$, i.e., $\bar{x}(s)=(1, x(s))$ and $\bar{y}(t)=$ $(1, y(t))$. Put $\tilde{\sigma}(s, t)=\sigma(\bar{x}(s), \bar{y}(t))$ as before.

The pull-back of the real part of the infinitesimal cross ratio is given by

$$
\left((x \times y)^{*} \mathfrak{R e} \Omega_{L}\right)(s, t)=\frac{\cos \theta_{L}(x(s), y(t))}{|x(s)-y(t)|^{2}}\left|x^{\prime}(s)\right|\left|y^{\prime}(t)\right| d s \wedge d t .
$$

On the other hand, the pull-back of the imaginary signed area element is given by

$$
\left((x \times y)^{*}\left(\left\langle\sigma_{x}, \sigma_{y}\right\rangle d x \wedge d y\right)\right)(s, t)=\left\langle\tilde{\sigma}_{s}, \tilde{\sigma}_{t}\right\rangle(s, t) d s \wedge d t .
$$

Fix any $\left(s_{0}, t_{0}\right)$. The Möbius invariance of the both sides allows us to assume that $x\left(s_{0}\right)$ and $y\left(t_{0}\right)$ are antipodal. Then, at $\left(s_{0}, t_{0}\right)$,

$$
\langle\bar{x}, \bar{x}\rangle=\langle\bar{y}, \bar{y}\rangle=0, \quad\langle\bar{x}, \bar{y}\rangle=-2 .
$$

Therefore, by the formula $(2)$, at $\left(s_{0}, t_{0}\right)$ there holds

$$
\langle p, p\rangle=4,\left\langle p, p_{s}\right\rangle=0,\left\langle p_{s}, p_{t}\right\rangle=-2 x^{\prime}\left(s_{0}\right) \cdot y^{\prime}\left(t_{0}\right),
$$

which implies

$$
\left\langle\tilde{\sigma}_{s}, \tilde{\sigma}_{t}\right\rangle=\frac{\langle p, p\rangle\left\langle p_{s}, p_{t}\right\rangle-\left\langle p, p_{s}\right\rangle\left\langle p, p_{t}\right\rangle}{\langle p, p\rangle^{2}}=-\frac{1}{2} x^{\prime}\left(s_{0}\right) \cdot y^{\prime}\left(t_{0}\right) .
$$

Since $x_{0}=x\left(s_{0}\right)$ and $y_{0}=y\left(t_{0}\right)$ are antipodal, we have (Figure 1)

$$
\theta_{L}\left(x\left(s_{0}\right), y\left(t_{0}\right)\right)=\pi-\angle x^{\prime}\left(s_{0}\right) \cdot y^{\prime}\left(t_{0}\right) .
$$

It follows that

$$
\left\langle\tilde{\sigma}_{s}, \tilde{\sigma}_{t}\right\rangle\left(s_{0}, t_{0}\right)=-\frac{1}{2} x^{\prime}\left(s_{0}\right) \cdot y^{\prime}\left(t_{0}\right)=2 \frac{\left|x^{\prime}\left(s_{0}\right)\right|\left|y^{\prime}\left(t_{0}\right)\right|}{\left|x\left(s_{0}\right)-y\left(t_{0}\right)\right|^{2}} \cos \theta_{L}\left(x\left(s_{0}\right), y\left(t_{0}\right)\right),
$$

which implies that the right-hand sides of (9) and (10) coincide. 


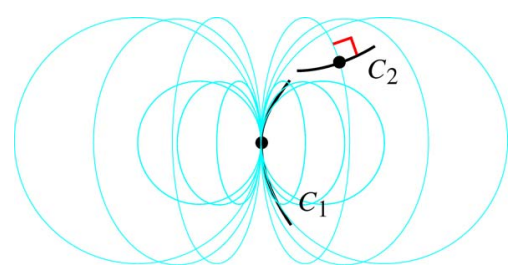

FIGURE 2. The circles of $\mathcal{C}_{x}$.

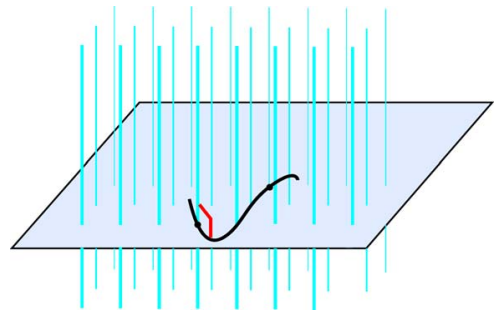

FIGURE 3. The image by $\pi$.

We remark that an alternative geometric proof can be obtained if we use pseudoorthonormal basis of $S^{3} \times S^{3} \backslash \Delta$ illustrated in Figure 10. This is because

$$
\left\langle\tilde{\sigma}_{s}+\tilde{\sigma}_{t}, \tilde{\sigma}_{s}+\tilde{\sigma}_{t}\right\rangle\left(s_{0}, t_{0}\right)=-x^{\prime}\left(s_{0}\right) \cdot y^{\prime}\left(t_{0}\right)
$$

implies $\left\langle\tilde{\sigma}_{s}, \tilde{\sigma}_{t}\right\rangle\left(s_{0}, t_{0}\right)=-(1 / 2) x^{\prime}\left(s_{0}\right) \cdot y^{\prime}\left(t_{0}\right)$.

COROLLARY 3.3. The imaginary signed area element of a product torus $C_{1} \times C_{2}$ with respect to the pseudo-Riemannian structure is equal to minus the pull-back of the canonical symplectic form:

$$
\left\langle\sigma_{x}, \sigma_{y}\right\rangle d x \wedge d y=-\iota^{*} \varphi^{*} \omega_{S^{3}} .
$$

This completes the proof of Theorem 2.2 (i).

We remark that a statement similar to that of the above corollary does not hold for a general surface in $S^{3} \times S^{3} \backslash \Delta$ as we will see in Subsection 4.3 in Appendix.

\subsection{Proof of Theorem 2.2 (ii). As}

$$
A\left(C_{1}, C_{2}\right)=2 \int_{C_{1} \times C_{2}} \frac{\left|\cos \theta_{L}(x, y)\right|}{|x-y|^{2}} d x d y,
$$

it is equal to 0 if and only if the conformal angle $\theta_{L}(x, y)$ is equal to $\pi / 2$ for any $x \in C_{1}$ and $y \in C_{2}$.

Suppose $A\left(C_{1}, C_{2}\right)=0$. Let $x$ be a point in $C_{1}$. Let $\mathcal{C}_{x}$ be the set of the circles which are tangent to $C_{1}$ at $x$. Then $C_{2}$ can intersect circles in $\mathcal{C}_{x}$ only at a right angle (Figure 2). Consider a stereographic projection $\pi$ from $S^{3} \backslash\{x\}$ to $\boldsymbol{R}^{3}$. It maps $\mathcal{C}_{x}$ to the set of parallel lines. Since $\pi\left(C_{2}\right)$ can intersect lines of $\pi\left(\mathcal{C}_{x}\right)$ only at a right angle, $\pi\left(C_{2}\right)$ is contained in a 2-plane which is orthogonal to the lines in $\pi\left(C_{x}\right)$ (Figure 3). Therefore, $C_{2}$ is contained in a sphere $\Sigma_{x}$ which intersects $C_{1}$ at a right angle at $x$ (Figure 4).

Let $x^{\prime}$ be a point of $C_{1}$ close to $x$. As $C_{1}$ intersects $\Sigma_{x}$ orthogonally at $x$, we can take $x^{\prime}$ outside $\Sigma_{x}$. Therefore, $\Sigma_{x} \neq \Sigma_{x^{\prime}}$. Since $C_{2}$ is contained in the intersection $\Sigma_{x} \cap \Sigma_{x^{\prime}}, C_{2}$ must be a circle (Figure 5). The same argument shows that $C_{1}$ is also a circle.

Consider the stereographic projection $\pi$ again. Since $C_{1}$ is a circle, $\pi\left(C_{1}\right)$ is a line. Then $\pi\left(C_{2}\right)$ is the intersection of two spheres which intersect the line $\pi\left(C_{1}\right)$ at a right angle (Figure $6)$. Therefore, $\pi\left(C_{2}\right)$ is symmetric in the line $\pi\left(C_{1}\right)$. It follows that $\pi\left(C_{1}\right) \cup \pi\left(C_{2}\right)$ is an image of the standard Hopf link (Figure 7). 


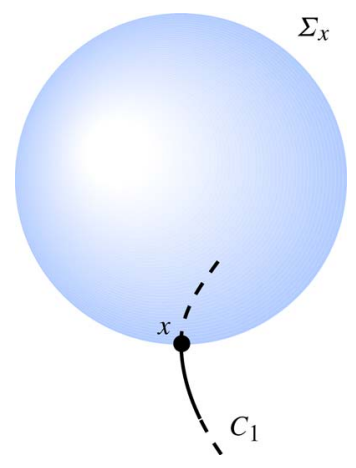

FIGURE 4.

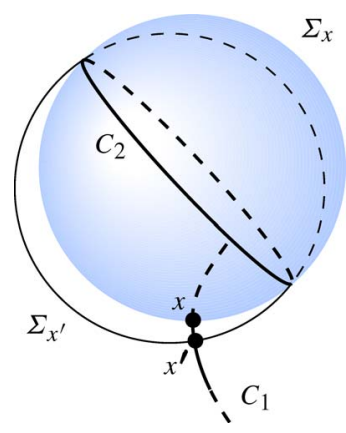

FIGURE 5.

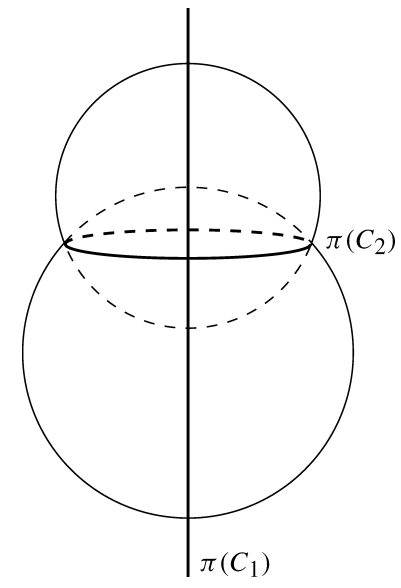

FIGURE 6.

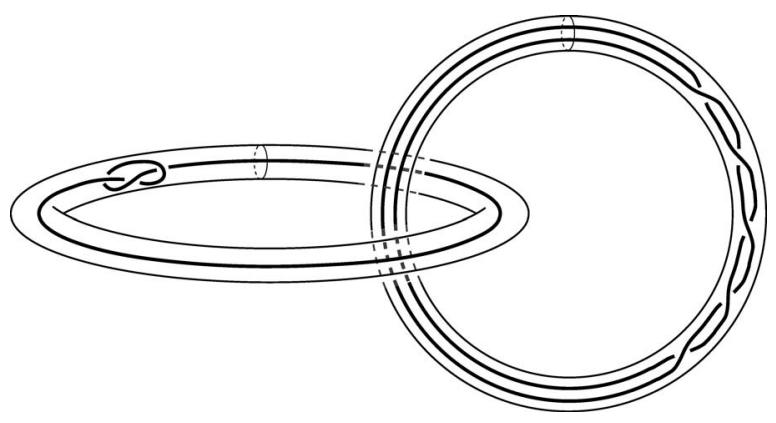

FIGURE 7. A satellite link of a Hopf link.

This completes the proof of Theorem 2.2 (ii).

3.4. Corollary and Conjecture. Let $[L]$ denote an isotopy class of a link $L$. Define

$$
A([L])=\inf _{C_{1}^{\prime} \cup C_{2}^{\prime} \in[L]} A\left(C_{1}^{\prime}, C_{2}^{\prime}\right) .
$$

COROLlary 3.4. If $L$ is a separable link or a satellite link of a Hopf link, then Area $([L])=0$.

Proof. Suppose $L=C_{1} \cup C_{2}$ is a separable link in $\boldsymbol{R}^{3}$. We can make $|x-y|(x \in$ $C_{1}, y \in C_{2}$ ) as big as we like. Now the conclusion follows from the formula (11).

Suppose $L=C_{1} \cup C_{2}$ is a satellite link of a Hopf link. Then, after an ambient isotopy, it can be contained in a very thin tubular neighbourhood of the standard Hopf link given by (5). Furthermore, for any positive constants $\delta_{1}$ and $\delta_{2}$, the link can be placed so that, outside 
a small region of $C_{1} \times C_{2}$ whose measure is $\delta_{1}$ Length $\left(C_{1}\right) \cdot \operatorname{Length}\left(C_{2}\right)$, the conformal angle satisfies $\left|\theta_{L}-\pi / 2\right| \leq \delta_{2}$. Then the formula (11) implies the assertion of the corollary since $|x-y|\left(x \in C_{1}, y \in C_{2}\right)$ is bounded below.

Conjecture 3.5. We conjecture that $A([L])$ does not always vanish. For example, if $L=C_{1} \cup C_{2}$ is a hyperbolic link each component of which is a non-trivial knot, then there is no solid torus $H_{1}$ so that $C_{1}$ is contained in $H_{1}$ and $C_{2}$ in $\boldsymbol{R}^{2} \backslash H_{1}$. We conjecture that $A([L])$ is positive for such a link type.

\section{Appendix.}

\subsection{Diagonal Möbius invariance characterizes $\omega$.}

PROPOSITION 4.1. Suppose $\rho$ is a 2-form on $S^{n} \times S^{n} \backslash \Delta$ which is invariant under the diagonal action of orientation preserving Möbius transformations. Then $\rho=c \omega$ for some constant $c$ if $n \neq 1$, where $\omega$ is the pull-back of the canonical symplectic form of $T^{*} S^{n}$ by the bijection from $S^{n} \times S^{n} \backslash \Delta$ to $T^{*} S^{n}$ given by (1), and $\rho=c_{1} \omega+c_{2} \mathfrak{I m}\left(d z \wedge d w /(w-z)^{2}\right)$ under the identification $S^{2} \cong \boldsymbol{C} \cup\{\infty\}$ for some $c_{1}, c_{2} \in \boldsymbol{R}$ if $n=2$.

This fact has been mentioned in [10] in a more general form (see $\S 3.2$ ). We put the proof here since the author could not find it in the literature.

PROOF. Since $S^{n} \times S^{n} \backslash \Delta$ is a homogeneous space of the Möbius group, it suffices to show the statement for $\rho$ restricted to a point $(x,-x)$ and the action of its isotropy group $H_{(x,-x)}$.

We may assume, without loss of generality, that $x$ and $-x$ correspond to $(1,1,0, \ldots, 0)$ and $-x=(1,-1,0, \ldots, 0)$ in the Minkowski space $\boldsymbol{R}_{1}^{n+2}$, respectively. Suppose an orientation preserving Möbius transformation $T_{A} \in H_{(x,-x)}$ is given by $A \in S O(n+1,1)$. Then, since $A$ keeps both $\operatorname{Span}\left\langle e_{0}, e_{1}\right\rangle$ and $\operatorname{Span}\left\langle e_{2}, \ldots, e_{n+1}\right\rangle$ invariant, $A$ can be expressed as

$$
A=\left(\begin{array}{cc}
A_{1} & O \\
O & A_{2}
\end{array}\right)
$$

where $A_{1} \in S O(1,1)$ and $A_{2} \in S O(n)$, or $A_{1} \in O(1,1) \backslash S O(1,1)$ and $A_{2} \in O(n) \backslash S O(n)$. However, in the latter case we have $T_{A}(x)=-x$, which does not fit the assumption that $T_{A}$ belongs to the isotropy group $H_{(x,-x)}$. It follows that the action of an element of $H_{(x,-x)}$ on

$$
T_{(x,-x)}\left(S^{n} \times S^{n} \backslash \Delta\right)=T_{x} S^{n} \oplus T_{-x} S^{n} \cong \boldsymbol{R}^{n} \oplus \boldsymbol{R}^{n}
$$

is generated by

$$
\begin{array}{llrl}
(u, v) & \mapsto\left(c u, c^{-1} v\right) & & \left(c \in \boldsymbol{R}^{\times}\right), \\
(u, v) \mapsto(g u, g v) & & (g \in S O(n)) .
\end{array}
$$

Thus, it is enough to give $\rho\left(e_{2}, v\right)\left(e_{2} \in T_{x} S^{n}, v \in T_{-x} S^{n}\right)$ to determine $\rho$.

(i) Suppse $n \geq 3$. Let $R$ be a 180 degree rotation of $\boldsymbol{R}^{n}=\operatorname{Span}\left\langle e_{2}, \ldots, e_{n+1}\right\rangle$ around $x_{2}$-axis. Then, since $\omega\left(e_{2}, v\right)=(1 / 2) v \cdot e_{2}$, we have

$$
\rho\left(e_{2}, v\right)=\frac{1}{2}\left(\rho\left(e_{2}, v\right)+\rho\left(e_{2}, R v\right)\right)=\rho\left(e_{2}, \frac{v+R v}{2}\right)
$$



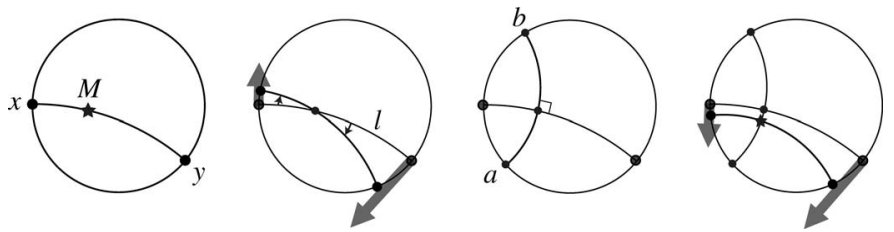

FIGURE 8. Pseudo-orthogonal basis of a tangent space of $S^{1} \times S^{1} \backslash \Delta$. A picture in Poincaré disc model.

$$
\begin{aligned}
& =\rho\left(e_{2},\left(v \cdot e_{2}\right) e_{2}\right)=\left(v \cdot e_{2}\right) \rho\left(e_{2}, e_{2}\right) \\
& =2 \rho\left(e_{2}, e_{2}\right) \omega_{S^{n}}\left(e_{2}, v\right),
\end{aligned}
$$

which implies $\rho=2 \rho\left(e_{2}, e_{2}\right) \omega$.

(ii) When $n=1$, the equation (13) also holds, which implies $\rho=2 \rho\left(e_{2}, e_{2}\right) \omega$.

(iii) Suppose $n=2$. Then

$$
\begin{aligned}
\rho\left(e_{2}, v\right) & =\left(v \cdot e_{2}\right) \rho\left(e_{2}, e_{2}\right)+\left(v \cdot e_{3}\right) \rho\left(e_{2}, e_{3}\right) \\
& =2 \rho\left(e_{2}, e_{2}\right) \omega\left(e_{2}, v\right)+4 \rho\left(e_{2}, e_{3}\right) \Im \mathfrak{m}\left(d z \wedge d w /(w-z)^{2}\right)\left(e_{2}, v\right),
\end{aligned}
$$

which implies $\rho=2 \rho\left(e_{2}, e_{2}\right) \omega+4 \rho\left(e_{2}, e_{3}\right) \mathfrak{I m}\left(d z \wedge d w /(w-z)^{2}\right)$.

It follows that the imaginary part of $d z \wedge d w /(w-z)^{2}$ cannot be generalized to $S^{n} \times S^{n} \backslash \Delta$ when $n \geq 3$. In fact, it can naturally be generalized to a Kähler form on $S O(n+1,1) / S O(2) \times$ $S O(n-1,1)$, which is the space of oriented codimension 2 spheres in $S^{n}$.

4.2. Pseudo-orthogonal basis of $S^{3} \times S^{3} \backslash \Delta$. Let us start with a baby case $S^{1} \times$ $S^{1} \backslash \Delta$. It can be identified with the set of oriented time-like planes in the 3-dimensional Minkowski space $\boldsymbol{R}_{1}^{3}$. By taking a positive unit normal vector to each of these planes, $S^{1} \times$ $S^{1} \backslash \Delta$ can be identified with the 2-dimensional de Sitter space $\Lambda=\left\{x \in \boldsymbol{R}_{1}^{3} ;\langle x, x\rangle=1\right\}$. Let $\Sigma=\{x, y\}$ be a pair of points in $S^{1} \cong \partial \boldsymbol{H}^{2}$. Let $l$ denote the geodesic in $\boldsymbol{H}^{2}$ which joins $x$ and $y$. Take a point $M$ on $l$ (Figure 8), then it determines two pencils as follows.

Let $a$ and $b$ be the "end points" of the geodesic in $\boldsymbol{H}^{2}$ which is orthogonal to $l$ at point $M$ (the third of Figure 8). Let $\mathcal{P}_{+}$be a pencil obtained by rotating the geodesic $l$ around $M$ and $\mathcal{P}_{-}$the Poncelet pencil with limit points $a$ and $b$. Then $\mathcal{P}_{+}$and $\mathcal{P}_{-}$can be considered as geodesics in $\Lambda$, namely, the intersections with $\Lambda$ and space-like and time-like 2-planes $\Pi_{ \pm}$. A pair of the unit tangent vectors to $\mathcal{P}_{+}$and $\mathcal{P}_{-}$at $\sigma$ can serve as a pseudo-orthonormal basis of $T_{\sigma} \Lambda$, where $\sigma$ is a point in $\Lambda$ that corresponds to $\Sigma$. These vectors can be obtained in $\Pi_{ \pm}$by rotation and Lorentz boost (hyperbolic rotation) of $\sigma$. The corresponding vectors in $S^{1} \times S^{1} \backslash \Delta$ are illustrated as the second and the last of Figure 8

Suppose $\{u, v\}$ is a pseudo-orthonormal basis of $T_{\sigma} \Lambda$. Then we have another basis, $\{(u+v) / \sqrt{2},(u-v) / \sqrt{2}\}$ consisting of two light-like vectors (Figure 9). This illustrates why $\sigma_{x}$ and $\sigma_{y}$ in Subsection 2.3 are null vectors. 

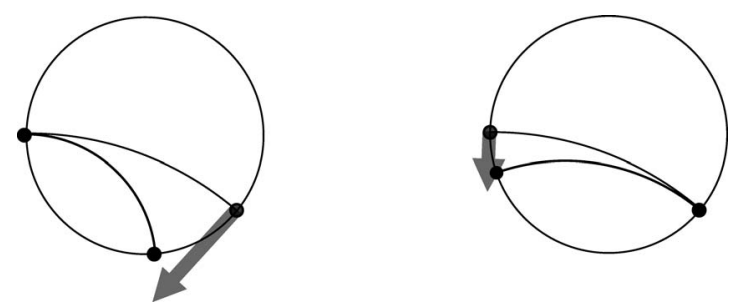

FIgURE 9. Light-like basis of a tangent space of $S^{1} \times S^{1} \backslash \Delta$. A picture in Poincaré disc model.

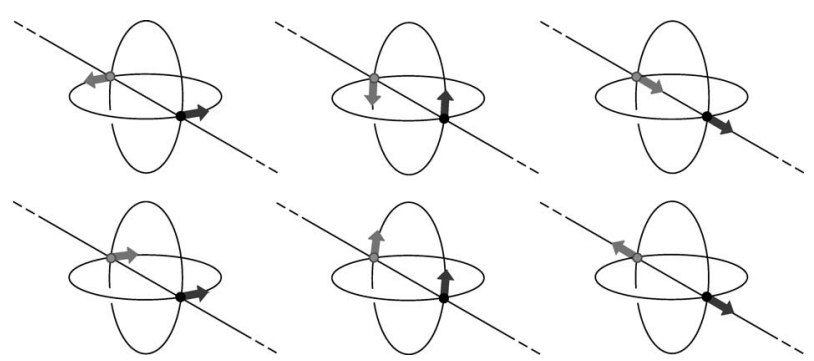

FIgURE 10. Pseudo-orthogonal basis of a tangent space of $S^{3} \times S^{3} \backslash \Delta$. Space-like vectors above and time-like vectors below. A picture in $\boldsymbol{R}^{3}$ obtained through a stereographic projection.

The pseudo-orthonormal basis of $S^{3} \times S^{3} \backslash \Delta$ can be given by that of $S^{1} \times S^{1} \backslash \Delta$. In fact, we can consider three mutually orthogonal circles through a given pair of points, and take a pseudo-orthonormal basis in each circle as illustrated in Figure 10.

4.3. The imaginary signed area element and the symplectic form. Corollary 3.3 does not necessarily hold for a surface in $S^{3} \times S^{3} \backslash \Delta$ which is not the product of two curves in $S^{3}$. Let us show it in $\boldsymbol{R}^{3} \times \boldsymbol{R}^{3} \backslash \Delta$, fixing a stereographic projection $p$ from $S^{3}$ to $\boldsymbol{R}^{3} \cup\{\infty\}$.

Suppose a pair of points in $\boldsymbol{R}^{3}$ are expressed by $X(s, t)$ and $Y(s, t)$. Let $M$ be a surface $\{(X(s, t), Y(s, t))\}_{(s, t) \in D}$ in $\boldsymbol{R}^{3} \times \boldsymbol{R}^{3} \backslash \Delta$, where $D$ is a domain in $\boldsymbol{R}^{2}$. Put $X_{s}=\partial X / \partial s, X_{t}=$ $\partial X / \partial t$, and

$$
\tilde{X}_{s}=2\left(X_{s}, \frac{X-Y}{|X-Y|}\right) \frac{X-Y}{|X-Y|}-X_{s}, \tilde{X}_{t}=2\left(X_{t}, \frac{X-Y}{|X-Y|}\right) \frac{X-Y}{|X-Y|}-X_{t} .
$$

Then $\widetilde{X}_{S}$ is the tangent vector at $Y$ to a circle which is tangent to $X_{S}$ at $X$ that passes through $Y$ with $\left|\widetilde{X}_{S}\right|=\left|X_{S}\right|$. The same interpretation also holds for $\tilde{X}_{t}$.

Note that the pull-back $\omega_{R^{3}}$ of the symplectic form $\omega$ introduced in Subsection 1.1 by a map $p^{-1} \times p^{-1}$ from $\boldsymbol{R}^{3} \times \boldsymbol{R}^{3} \backslash \Delta$ to $S^{3} \times S^{3} \backslash \Delta$, where $p$ is a stereographic projection from 
$S^{3}$ minus one point to $\boldsymbol{R}^{3}$, is given by

$$
\omega_{\boldsymbol{R}^{3}}=2\left(\frac{\sum_{i=1}^{3} d X_{i} \wedge d Y_{i}}{|X-Y|^{2}}-2 \frac{\left(\sum_{i=1}^{3}\left(X_{i}-Y_{i}\right) d X_{i}\right) \wedge\left(\sum_{j=1}^{3}\left(X_{j}-Y_{j}\right) d Y_{j}\right)}{|X-Y|^{4}}\right)
$$

(see [12]). The pull-back of $\omega_{\boldsymbol{R}^{3}}$ by the map $X \times Y$ from $D$ to $\boldsymbol{R}^{3} \times \boldsymbol{R}^{3} \backslash \Delta$ is given by

$$
(X \times Y)^{*} \omega_{\boldsymbol{R}^{3}}=-2\left(\widetilde{X}_{s} \cdot Y_{t}-\tilde{X}_{t} \cdot Y_{s}\right) \frac{d s \wedge d t}{|X-Y|^{2}} .
$$

This can be verified by showing that the both sides coincide when $X$ and $Y$ are located on specific positions, say $X\left(s_{0}, t_{0}\right)=(1,0,0)$ and $Y\left(s_{0}, t_{0}\right)=(-1,0,0)$ because the both sides are equivariant under the diagonal action of Möbius transformations.

On the other hand, the "signed area element" $\alpha_{M}$ of $M$ associated with the pseudoRiemannian structure of $\Theta(0,3)$ can be given as follows. Let $\hat{\sigma}$ be the composite

$$
\hat{\sigma}: D \stackrel{X \times Y}{\longrightarrow} M \hookrightarrow \boldsymbol{R}^{3} \times \boldsymbol{R}^{3} \backslash \Delta \stackrel{p^{-1} \times p^{-1}}{\longrightarrow} S^{3} \times S^{3} \backslash \Delta \stackrel{\cong}{\stackrel{\cong}{\longrightarrow}} \Theta(0,3) .
$$

Using the pseudo-orthonormal basis illustrated in Figure 10 and the Möbius invariance, we have

$$
\begin{aligned}
(X \times Y)^{*} \alpha_{M} & =\sqrt{\operatorname{det}\left(\begin{array}{cc}
\left\langle\hat{\sigma}_{s}, \hat{\sigma}_{s}\right\rangle & \left\langle\hat{\sigma}_{s}, \hat{\sigma}_{t}\right\rangle \\
\left\langle\hat{\sigma}_{t}, \hat{\sigma}_{s}\right\rangle & \left\langle\hat{\sigma}_{t}, \hat{\sigma}_{t}\right\rangle
\end{array}\right)} d s \wedge d t \\
& =2 \sqrt{\operatorname{det}\left(\begin{array}{cc}
2 \widetilde{X}_{s} \cdot Y_{s} & \left.\widetilde{X}_{s} \cdot Y_{t}+\widetilde{X}_{t} \cdot Y_{s}\right) \\
\widetilde{X}_{s} \cdot Y_{t}+\widetilde{X}_{t} \cdot Y_{s} & 2 \widetilde{X}_{t} \cdot Y_{t}
\end{array}\right)} \frac{d s \wedge d t}{|X-Y|^{2}} .
\end{aligned}
$$

Therefore, the imaginary signed area element $\sqrt{-1} \alpha_{M}$ coincides with the pull-back of the canonical symplectic form $\left.\omega_{R^{3}}\right|_{C_{1} \times C_{2}}$ up to sign if and only if $\left(\tilde{X}_{s} \cdot Y_{t}\right)\left(\tilde{X}_{t} \cdot Y_{s}\right)=$ $\left(\widetilde{X}_{s} \cdot Y_{s}\right)\left(\widetilde{X}_{t} \cdot Y_{t}\right)$, which holds if and only if $\widetilde{X}_{s} \times \widetilde{X}_{t} \perp Y_{s} \times Y_{t}$. It does not always hold in general.

We remark that this condition does not necessarily imply that the surface is a product of two curves. We also remark that the above condition is always satisfied for a surface in $S^{1} \times S^{1} \backslash \Delta$.

4.4. Remark on energy minimizing Hopf links. There is another variational characterization of the "best" Hopf link.

The Möbius cross energy [7] of a 2-component link $C_{1} \cup C_{2}$, which is generalzation of the energy for knots defined by the author [14], is given by

$$
E\left(C_{1}, C_{2}\right)=\int_{C_{1} \times C_{2}} \frac{d x d y}{|x-y|^{2}}
$$

This energy is also invariant under Möbius transformations. Recently, Agol, Marques and Neves proved Freedman-He-Wang's conjecture, namely, they showed that if the linking number of $C_{1}$ and $C_{2}$ is equal to \pm 1 , then $E\left(C_{1}, C_{2}\right) \geq 2 \pi^{2}$, and that the equality holds if and only if $C_{1} \cup C_{2}$ is an image of the "best" Hopf link by a Möbius transformation. This is a much more difficult problem, and was proved using min-max theory which has also been used in the proof of the Willmore conjecture [13]. 
The formula (11) implies $E\left(C_{1}, C_{2}\right) \geq(1 / 2) A\left(C_{1}, C_{2}\right)$. To be more precise, the equality does not occur since the conformal angle between different components of a link cannot be identically zero. It might be interesting to point out that the infimum of $A\left(C_{1}, C_{2}\right)$ over all the 2-component links is attained not at trivial links, but at the "best" Hopf link and the conformal image of it, whereas the infimum of $E\left(C_{1}, C_{2}\right)$ over all the 2-component links is not attained, as $E\left(C_{1}, C_{2}\right)$ tends to +0 as the distance between $C_{1}$ and $C_{2}$ tends to $+\infty$.

\section{REFERENCES}

[1] I. Agol, F. C. Marques And A.Neves, Min-max theory and the energy of links, arXiv:1205.0825.

[2] L. V. Ahlfors, Cross-ratios and Schwarzian derivatives in $\boldsymbol{R}^{n}$, Complex analysis, Birkhauser, Basel, 1988, Articles Dedicated to Albert Pfluger on the Occasion of his 80th Birthday, 1-15.

[ 3 ] A. BESSE, Manifolds all of whose geodesics are closed, Ergeb. Math. Grenzgeb. 93, Springer-Verlag, BerlinNew York, 1978.

[ 4 ] J. A. Calvo, K. C. Millett and E. J. Rawdon, Physical knots: knotting, linking, and folding geometric objects in $\boldsymbol{R}^{3}$ : Ams Special Session on Physical Knotting and Unknotting, Las Vegas, Nevada, April 21-22, 2001, Contemporary Mathematics 304, Amer Mathematical Society, Providence, 2002.

[ 5 ] J. A. Calvo, K. C. Millett, E. J. Rawdon and A. Stasiak eds., Physical and numerical models in knot theory: Including Applications To The Life Sciences, Series on Knots and Everything Vol. 36, World Scientific Publishing Co. Pte. Ltd., Singapore, 2005.

[6] E. FERrAnd, Sur la structure symplectique de l'espace des géodésiques d'un espace de Hadamard, Geom. Dedicata 68 (1997), 79-89.

[ 7 ] M. H. Freedman, Z-X. He And Z. WAng, Möbius energy of knots and unknots, Ann. of Math. 139 (1994), $1-50$.

[ 8 ] F. HÉLEIN, Isoperimetric inequalities and calibrations, "Progress in Partial Differential Equations: the Metz surveys", M. Chipot and I. Shafrir ed., Pitman Research Notes in Mathematics, Series 345, Longman (1996), 92-105.

[ 9 ] U. Hertrich-Jeromin, Introduction to Möbius Differential Geometry, London Math. Soc. Lecture Note Ser. 300, Cambridge University Press, Cambridge, 2003.

[10] M. KanaI, Differential-geometric studies on dynamics of geodesic and frame flows, Japan J. Math. 19 (1993), $1-30$.

[11] W. KLingenberg, Riemannian geometry, Walter de Gruyter, Berlin, 1982.

[12] R. LAngevin And J. O’HARA, Conformally invariant energies of knots, J. Institut Math. Jussieu 4 (2005), 219-280.

[13] F. C. Marques And A. Neves, Min-Max theory and the Willmore conjecture, arXiv:1202.6036.

[14] J. O'HARA, Energy of a knot, Topology 30 (1991), 241-247.

[15] J. O'HARA, Energy of knots and conformal geometry, Ser. Knots Everything 33, World Scientific Publishing Co., Inc., River Edge, NJ, 2003.

[16] A. Stasiak, V. Katritch And L. H. Kauffman eds., Ideal knots, Series on Knots and Everything Vol. 19, World Scientific, Singapore, 1998.

DEPARTMENT OF MATHEMATICS AND INFORMATION SCIENCES

TOKYO METROPOLITAN UNIVERSiTy

1-1 Minami-OHSAWA, HaChIOUJI-SHI

TOKYO 192-0397

JAPAN

E-mail address: ohara@tmu.ac.jp 